\title{
Activating NOTCH1 Mutation
}

National Cancer Institute

\section{Source}

National Cancer Institute. Activating NOTCH1 Mutation. NCI Thesaurus. Code C158063.

A change in the nucleotide sequence of the NOTCH1 gene that that results in constitutive activation of both neurogenic locus notch homolog protein 1 and its downstream signaling pathways. 